\title{
Does the lunar regolith contain secrets of the Solar System? Using the Moon as a cosmic witness plate
}

\author{
David S. McKay ${ }^{1}$, Louise Riofrio ${ }^{2}$ and Bonnie L. Cooper ${ }^{2}$ \\ ${ }^{1}$ NASA Johnson Space Center, Houston, Texas 77058, USA \\ ${ }^{2}$ Oceaneering Space Systems, NASA Johnson Space Center, Houston, Texas 77058, USA \\ email: L.M.Riofrio@nasa.gov
}

\begin{abstract}
The lunar regolith (soil) has recorded a history of the early Moon, the Earth, and the entire solar system. A major goal of the developing lunar exploration program should be to find and play back existing fragments of that tape. By playing back the lunar tape, we can uncover a record of planetary bombardment, as well as solar and stellar variability. The Moon can tell us much about our place in the solar system and in the Universe. The lunar regolith has likely recorded the original meteoritic bombardment of Earth and Moon, a violent cataclysm that may have peaked around $4 \mathrm{GY}$, and the less intense bombardment occurring since that time. Decrease in bombardment allowed life to develop on Earth. This impact history is preserved as megaregolith layers, ejecta layers, impact melt rocks, and ancient impact breccias. The impact history for the Earth and Moon possibly had profound effects on the origin and development of life. Life may have arrived via meteorite transport from a more quiet body, such as Mars. The solar system may have experienced bursts of severe radiation from the Sun, other stars or from unknown sources. The lunar regolith has also recorded a radiation history in the form of implanted and trapped solar wind and solar flare materials and radiation damage. The Moon can be considered as a giant tape recorder containing the history of the solar system. Lunar soil generated by small impacts will be found sandwiched between layers of basalt or pyroclastic deposits. This filling constitutes a buried time capsule that is likely to contain well-preserved ancient regolith. Study of such samples will show us how the solar system has evolved and changed over time. The lunar recording can provide detailed snapshots of specific portions of solar and stellar variability.
\end{abstract}

Keywords. Moon; meteors, meteoroids; solar system: formation

The Moon is a witness plate for solar system history. Humans have an almost genetic need to understand how we got here, and where we may be headed. Answers to important questions may be preserved in the lunar regolith. Fossil regolith is affected by meteorite impacts, impact exotic clasts, implant galactic cosmic rays, solar flares radiation and particles, and solar wind. New studies of the lunar regolith should have much broader implications than just understanding regolith formation.

Lunar soil generated by small impacts will be found sandwiched between layers of basalt, layers of pyroclastic deposits, layers of impact ejecta, layers of impact melt sheets, and various combinations of these deposits. This sandwich filling has been trapped and removed from further processing at many places. It constitutes a buried time capsule likely to contain records of solar wind, giant solar flares, and impacting bodies such as comets or asteroids. This buried regolith may also a record of major, but rare events such as giant cosmic ray bursts or greatly enhanced meteorite bombardment derived from the fragmentation of large asteroids. Study of such samples will show us how the 
solar system has evolved and changed over time and provide us with detailed snapshots of specific portions of solar system history.

While we should not expect Moon-wide pristine layers to be preserved, isolated fragments of such layers may be found in many places protected from destruction or gardening by rapid subsequent burial. Regolith samples from the Apollo collection began with scoop samples collected from depths up to $10 \mathrm{~cm}$. Trenches were dug to depths of $30 \mathrm{~cm}$. Drive tubes were dug to depths ranging from $17-65 \mathrm{~cm}$. The deepest samples were cores from depths ranging from 225-262 cm. The estimated time since burial of the trench samples ranged from 10 Myr to 100 Myr. Portions of the Apollo 15-17 drill cores had burial ages up to 1 Gyr.

However, much older regolith samples are within reach at appropriate lunar sites. Samples found beneath ejecta blankets of large craters may have burial or time capsule ages ranging up to 4 Gyr. Samples found beneath pyroclastic ash deposits have a lower limit exposure age defined by the underlying surface, and an upper limit given by the age of the overlying pyroclastic deposit. Based on Apollo data, typical ages range from 3.2-3.5 Gyr. Samples found between or beneath basalt flows have a lower and upper age ranges given by ages of the underlying and overlying basalt. These ages range from less than 3 to more than 4 Gyr. Similarly, samples located within breccia sheets in highland terrain have lower and upper age ranges corresponding to ages of the underlying and overlying breccia sheets. Ages of these breccia sheets range up to 4.4 Gyr. A key goal in our science of astrobiology is to determine whether life evolved independently on Earth. Some theories suggest that life arrived on Earth from elsewhere via meteorites. How long did planetary objects bombard the Moon and Earth during the early heavy bombardment? When did the rate of bombardment decrease, finally allowing life to catch hold on Earth? The impact history for the Earth, likely to be similar to that for the Moon, possibly had profound effects on the origin and development of life. The record of the first billion years has been destroyed on the Earth by geologic processing and resurfacing.

Did life come via meteorite transport from a quieter Mars? At the distance of Mars orbit, meteorite impacts were likely less severe and life could have started earlier. Mars life could become implanted on Earth via Martian meteorites, taking root in nutrient-rich oceans and ponds. The lunar regolith likely contains a record of the original bombardment earlier than $4 \mathrm{Gyr}$, the violent lunar cataclysm that may have peaked around $4 \mathrm{Gyr}$, as well as the less intense bombardment occurring since that time. Undamaged meteorite fragments have already been found on the Moon. Two examples were found at Station 9 in Hadley Rille by Apollo 15 astronauts and at Bench Crater by Apollo 12 astronauts. Many more fragments may be found in ancient regolith. The potential exists that fragments of Martian meteorites, Earth-derived meteorites, and even meteorites from moons of Jupiter and Saturn. Meteorites from Venus may also be buried in lunar regolith. During possible future mining operations to support lunar resource extraction and processing, a simple additional screening step might be added which could robotically detect non-lunar composition rocks and collect them for detailed examination. For example, based on the abundance of $\mathrm{Na}$ alone, it is possible to distinguish many Earth rocks from lunar rocks using a cutoff of about $0.5 \mathrm{Na}_{2} \mathrm{O} \%$. The potential exists that early meteorites from Earth found on the moon may preserve a record of the beginnings of life on Earth; these records are likely now destroyed on Earth by geologic processing, tectonics, and weathering.

Evolution of life would also be affected by radiation from space. In the course of its history, our solar system may have experienced strong bursts of severe radiation. Radiation might have come from the sun, other stars, supernovae, black holes, or other unknown sources. Severe radiation could blast away the ozone layer, causing major mutations in the development of life. Were these radiation bursts strong enough to kill early life on 
Earth? Will we be able to recover a record of them trapped in pockets of ancient lunar regolith?

Solar wind particles have energies up to $1 \mathrm{keV} /$ nucleon and penetrate the regolith to depths of 300 angstroms. More energetic solar electric protons (SEP) have energies from 1-100 $\mathrm{KeV}$ and can penetrate to depths of $1 \mathrm{~cm}$. Galactic cosmic rays can have energies from $100 \mathrm{KeV}$ to in excess of $3 \mathrm{GeV}$ and can penetrate $>1$ meter into the regolith. A record of these strong radiation bursts could be preserved in ancient regolith samples on the Moon. It is likely that unusual fluxes could be detected in time capsule regoliths.

Burial of existing regolith by a hot basalt flow, a hot impact-generated melt flow, or even an impact-produced base surge of debris could preserve ancient regolith. Similar flows are found on Earth in locations like Craters of the Moon National Park and many locations in Hawaii, and these flows override and preserve underlying soil horizons. Large areas of presumed pyroclastic dark mantle are found on the front side of the moon, and small dark mantle regions have been identified on the back side. The contact between dark mantle and underlying normal regolith may be less disturbed than the contact between a basalt flow and underlying regolith and the dark mantle deposits may be less likely to heat and bake out the underlying regolith, thereby preserving the solar particulate profile and other details.

Plans are currently being made to return humans to the Moon by the early 2020's. Already a small armada of spacecraft from several nations has orbited the Moon. These orbiting spacecraft will soon be joined by automated landers. A major goal of the developing lunar exploration program should be to find and sample existing fragments of the Solar System record in the form of carefully located and sampled pockets of preserved ancient regolith. The lunar regolith is the only readily accessible place in the solar system for finding and revealing the detailed record of solar system history. The witness plate aspect of the moon may ultimately turn out to be its single most valuable scientific asset, and we need to focus a major exploration effort in this direction. This focus is readily understood by the public and can be the underlying scientific basis of much of our lunar program. The recovery, sample return and decoding of pristine ancient regolith may contribute to the most important scientific discoveries of the new lunar era. 